\title{
Application of Virtual Reality in Chemical Education Using Intermolecular Force Display System HaptiChem
}

\author{
Hiroko Satoh a, b,c *, Akira Narita d, Atsushi Aoki e, Katsuhito Akahane f, \\ Makoto Sato ${ }^{\mathrm{f}}$
}

a) Department of Chemistry, University of Zurich, Winterthurerstrasse 190, 8057 Zurich, Switzerland

b) Research Organization of Information and Systems (ROIS), Minato-ku, Tokyo 105-0001, Japan

c) National Institute of Informatics (NII), Chiyoda-ku, Tokyo 101-8430, Japan

d Tokyo Tech High School of Science and Technology, Tokyo 108-0023, Japan

e) Kyoto Sangyo University, Faculty of Information Science and Engineering, Kyoto 603-8555, Japan

f) Institute of Innovative Research, Tokyo Institute of Technology, Yokohama 226-8503, Japan

\begin{abstract}
Virtual reality technology has been recently more intensively applied in chemistry. HaptiChem, which was developed in 2006, is one of the systems appeared in the early stage of this field. It is an intermolecular force display system, which makes it possible to touch and move molecules as feeling intermolecular force in a three-dimensional virtual space by using a haptic device. The functions and graphic display were designed as simple as possible for educational use, so that learner can easily grasp the meaning of the concept of molecular forces. We introduced HaptiChem in chemical education. We held a high school chemistry class with 43 students entitled "Several Forces between Molecules" as being open to the media. The students learned about intermolecular force from a lecture together with experiences of the force using HaptiChem. They asked more questions about intermolecular force during the class than usual. Their answers to the questionnaires after the class indicated that the haptic system promoted curiosity and enhanced learning. The students could learn more effectively by combining with the active feeling with HaptiChem. The highschool teachers evaluated that such a system can more efficiently encourage students to learn and remember things by stimulating their sense of touch. The observations also suggested that a haptic device made it easier to establish three-dimensional perception, which is difficult only with 2D-display. This pilot experiment was performed on 15th March 2007. It was the first attempt at using it in the field of chemical education. The observation was done fourteen years ago. However, since the effectiveness of haptic device in chemical education has not been changed and the interests of the applications have been increased, we decided to report the data we observed, which should be still worth disclosing. We wish to dedicate the results to new developments now and in the future.
\end{abstract}

Keywords: Virtual reality, Haptic device, Molecular model, Intermolecular force, Visualization, Chemical education, Three-dimensional perception, HaptiChem, SPIDAR, ChemoJun

*hiroko.satoh@chem.uzh.ch 


\section{Introduction}

Visualization and modeling of chemical phenomena and concepts are essential for knowing, analyzing, and understanding things better as well as for establishing communication exchanges to share data and concepts in chemical research and education. For the last several decades, many computer graphics tools for drawing and visualizing chemical structures and properties have been generally used in both research and education. In chemical education, inducing curiosity of learners at the early stage is important. However, most of chemical concepts treating microscaled invisible phenomena are difficult to understand especially for beginners. Effective tools that can help learners to make image and to grasp the concepts are useful in this phase. This is reason why chemical educational textbooks and tools using color cartoons, pictures, and computer-graphics are nowadays common in actual educational scenes.

Conventional devices for human-computer interface (HCI) are display and mouse. Besides these basic interface tools for visualization and input, new devices with virtual reality (VR) technology have been developed. One of the VR interface is for haptization, which extend HCI not only with sense of sight and hearing but also with sense of touch and force. The haptic technology has been applied to various fields including surgical simulation.

Since early 1990s haptic molecular modeling systems have been reported [1-15]. To our best knowledge, the GROPE project started by B. Brooks et al in 1967 is the first one to introduce haptic technology to molecular modelling [1]. Many of the pioneering works are for docking study of large biomolecular systems $[1,2,5,6]$. Other pioneering systems are for feeling quantum mechanical force to an electron in a molecular system [3], for sensing force on electrostatic potential [4], and for virtual experience of molecular dynamics [5,8]. Stabilization of haptic molecular systems was also studied [7]. Those systems use a haptic device called PHANTOM [16], which is a force display with parts like an arm with pencil. HaptiChem [8] is one of the systems at the early stage for haptic experience in chemistry. It is an intermolecular force display system, which makes it possible to touch and move molecules as feeling intermolecular force in a threedimensional virtual space by using a haptic device. HaptiChem uses a force display device SPIDAR [17], which was developed by Sato et al.

A new wave of haptic experience in chemistry has come together with a boom of artificial intelligence (AI) technology. Several elaborate haptic molecular modeling systems with the help of advanced computer technologies have been intensively studied for exploration of chemical reactions on quantum mechanical potential energy surfaces (PES) $[9,10,12,13,15]$ and for interactive MD [14]. These systems use PHANTOM as a haptic device. Some GROPE-like systems using VR interface without haptization have been also reported for exploration of PES [18] and 
molecular docking [19]. Such systems can gain haptization if it is combined with modern haptic devices like SPIDAR-W [20]. An idea of organic haptic device for materials design [21] is also expected to widen the possibilities of haptic molecular systems.

HaptiChem was developed by some of the authors for chemical research and education. It was designed in the framework of human-centered interaction with computer for enhancing research and learning activities [22-23]. After implementing fundamental functions to the system and having demonstrations in conferences for chemical educations [24-26], we conducted a pilot experiment at a high school by holding a chemistry class entitled "Several Forces between Molecules" as being open to the media in 2007. It was the first attempt at using it in the field of chemical education. The observations from the experiment suggested that the haptic system promoted curiosity and enhanced learning. The students could learn more effectively by combining with the active feeling with the haptic experiences. A haptic device made it easier to establish three-dimensional perception, which is difficult only with 2D-display. The high-school teachers evaluated that such a system can more efficiently encourage students to learn and remember things by stimulating their sense of touch. The outline of this pilot experiment was reported by the press [27-30], the high school [31], and some of the authors [32]. However, the details of the results have not yet been reported. The effectiveness of haptic device in chemical education has not been changed, and the interests of the applications have been increased [33, 34]. Although the experiment was held fourteen years ago, we believe that the data we observed is still worth disclosing. Therefore, we report here the details of the observations as well as the overview of the HaptiChem system.

\section{HaptiChem}

HaptiChem is an educational and research tool for virtually feeling molecular interactions by combining visualization and haptic technologies [8]. The concept of the system is to provide virtual experience of touching molecules and feeling the force between them to help learner to get image and to grasp the concept of molecules, which is the most fundamental issue in chemistry.

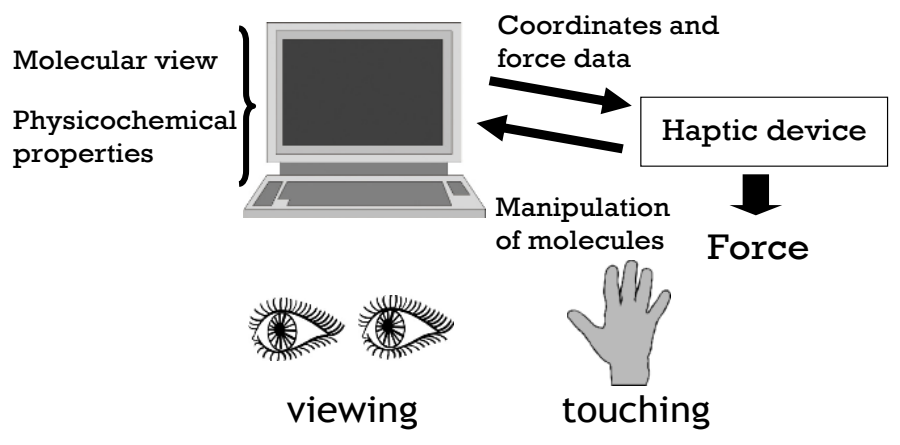

Figure 1. Design of HaptiChem 


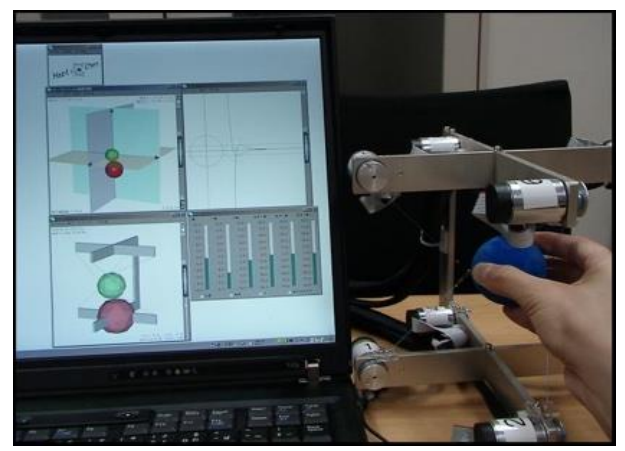

Figure 2. HaptiChem: Graphic display with haptic device for force display

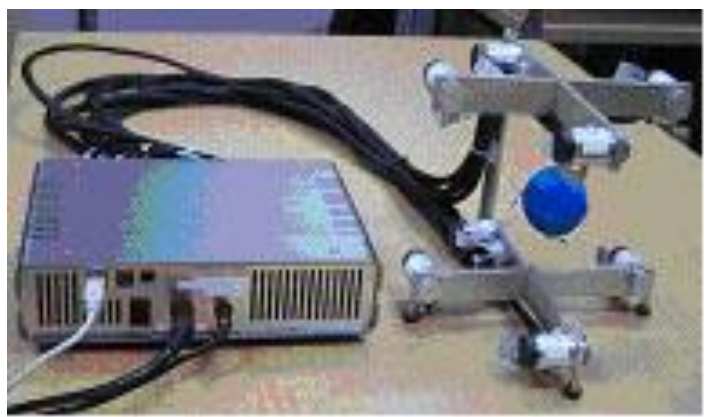

Figure 3. Haptic device SPIDAR-G

Figures 1 and 2 show the design and a photo of HaptiChem, respectively. A movie of operating HaptiChem is also published [35]. Molecules with physicochemical parameters are visualized on the graphic display. The haptic device enables a three-dimensional (3D) mouse to display force. The user can move one of the molecules in the 3D space by using the 3D mouse, while physically feeling the intermolecular force in the distance between the molecules. User can recognize the physicochemical parameters and the magnitude of the forces displayed on the 3D mouse at the same time. The haptic device used here is called SPIDAR-G [36] (Figure 3), which was developed by Sato et al. This is a tension based 7-DOF force feedback device with a sphere grip. The current shape of SPIDAR-G can be seen at a website of ArachnoForce Inc. $[37,38]$ The visualization software was developed based on a chemical graphics library ChemoJun $[39,40]$ and a multimedia graphics library Jun [41]. The basic requirements for a molecular haptic system were examined via experiments in the previous paper on HaptiChem [8]. 
(a)

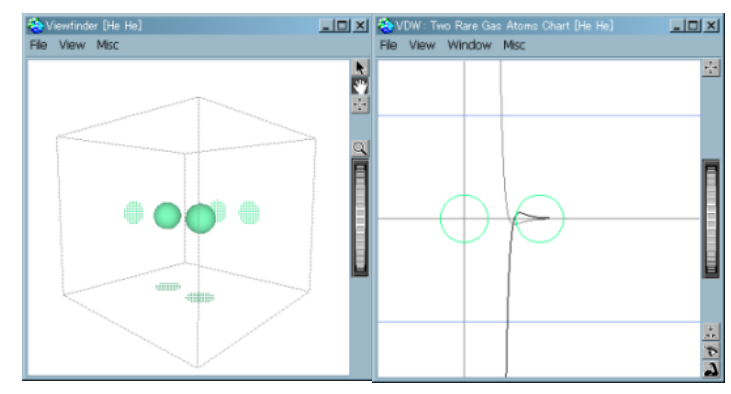

(b)

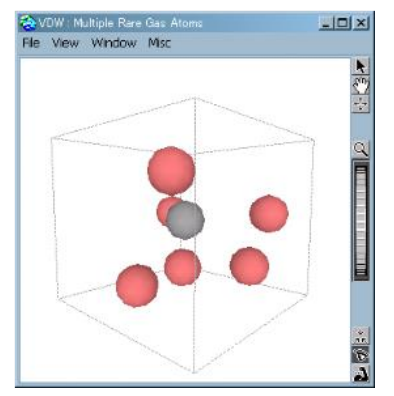

Figure 4. Display of intermolecular force between two molecules (a) and from multiple molecules (b)

HaptiChem has two functions for feeling the intermolecular force for rare gas atoms $(\mathrm{He}, \mathrm{Ne}$, $\mathrm{Ar}, \mathrm{Kr}$, and Xe). One is the intermolecular force between two atoms (Figure 4a). A potential and force functions chart are drawn on the display, and the indicator pointing to the distance between the molecules simultaneously moves as user moves the molecule. User can thus better understand the relationships between the curve of functions and the changes of force. The other function is to feel the intermolecular force from multiple molecules (Figure $4 \mathrm{~b}$ ). The number of molecules and speed of motion can be changed. The user can feel the differences of states by changing them. We designed functions and graphic display as simple as possible for educational use, so that learner can easily grasp the meaning of the concept of molecular forces. The stability is also important to be used in schools. The processing speed became faster compared to the first version [8] by improving the algorithm, so as to enhance stability of manipulation.

HaptiChem is freeware and open to public since 2007 [42, 43]. Whether or not a license for Cincom Smalltalk is required depends on the purpose of use [44].

\section{Chemistry Class with HaptiChem in High School}

\subsection{Procedure}

To better understand how effective HaptiChem could be in the field of chemical education, we examined the efficiency of HaptiChem in chemical education. On 15 $5^{\text {th }}$ March 2007, we conducted an experiment for using the haptic system in teaching chemistry at Tokyo Tech High School of Science and Technology. We held a chemistry class with 43 students entitled "Several Forces between Molecules". The class was open to the media.

The first half of the class about for 50 minutes was a lecture on the intermolecular forces from both organic and physical chemistry aspects. The teacher gave a lecture on what kinds of intermolecular forces are known in chemistry, what the physical bases of the forces are, and how the forces play important roles in chemical phenomena, by using a projector and a white board. The latter half of the class, about $60 \mathrm{~min}$., was used to experience HaptiChem firsthand for every 
student. It took about 1 min. per person. After the experience, each student answered a written questionnaire. Figure 5 shows photographs of the environment in which the lecture and the students' HaptiChem experiencing took place, which were taken in the chemical class. The reports by the press told the story of this pilot experiment [27-30].
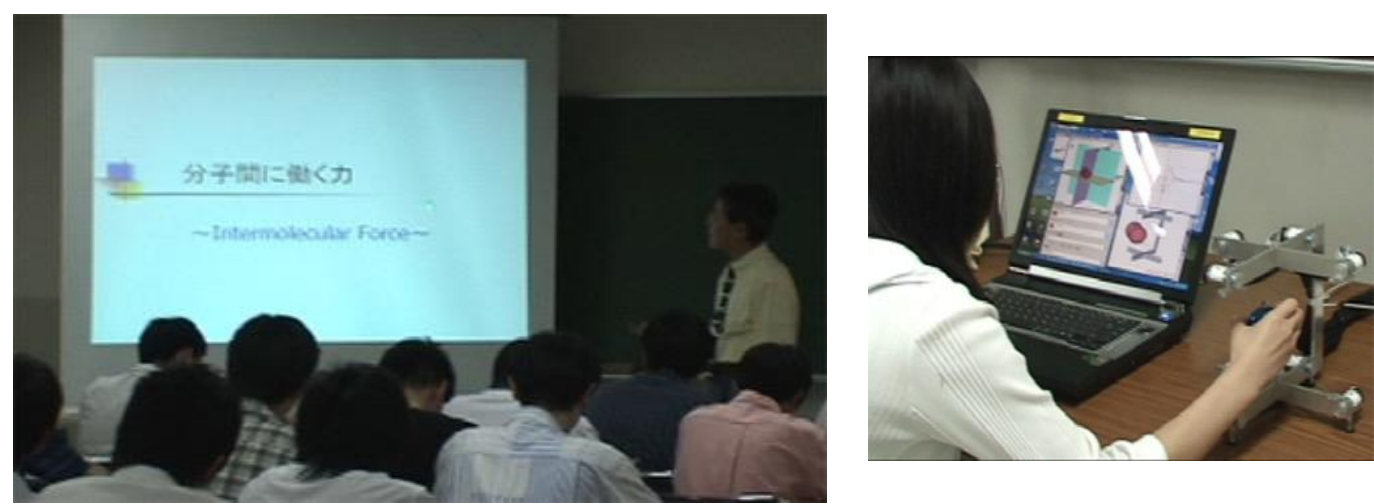

Figure 5. Lecture and a student experiencing HaptiChem

\subsection{Profile of Students}

The students were around 16 years old. They belonged to an applied chemistry course of the high school, thus most of the students liked chemistry. About $60 \%$ of the students answered that they liked chemistry very much or liked chemistry, and about $30 \%$ answered that they basically liked it. About $95 \%$ of the students answered that chemistry is one of their top three favorite subjects concerning the natural sciences, chemistry, physics, biology, geosciences, and mathematics. About $63 \%$ expressed that they wanted to continue studying chemistry as a major subject after entering a university.

\subsection{Results of Experiments}

Almost all the students were clearly excited at the moment that they felt the force from the 3D mouse. Some of the students were forced to think very deeply as they were feeling the force; they seemed to be reconsidering what they had just learned in the lecture.

After the experience, questions about the intermolecular force were raised by some of the students, such as "In the case of ions and other molecules, is the force different from that for the rare gas atoms? How about them?" and "What about the force in chemical reactions?". As they were asking, they seemed to be imagining what kind of feeling they might experience if it were possible.

The teachers evaluated that not only listening to the lecture but also having experienced it through the sense of touch was more impressive and encouraged the students to remember the lecture in more defined detail. It was also pointed out that the haptic system enabled the user to 
more easily establish a three-dimensional perception of something, which is difficult to make using only a 2D-viewer.

The results show that the experience stimulated students to think more deeply and encouraged them to study out of more curiosity so as to extend their scientific imagination.

\subsection{Answers to Questionnaires}

Answers to the questionnaires were obtained from 38 students after the experience. Figures 6 and 7 show the results.

For questionnaires on the lecture, about $92 \%$ of the students answered that the lecture combined with the HaptiChem system was exciting (Figure 6-1), and about 45\% felt that the time for experimentation was short (Figure 6-2). About 63\% answered that they could understand the intermolecular force (Figure 6-3), and about 89\% expected to use HaptiChem in the future (Figure 6-4).

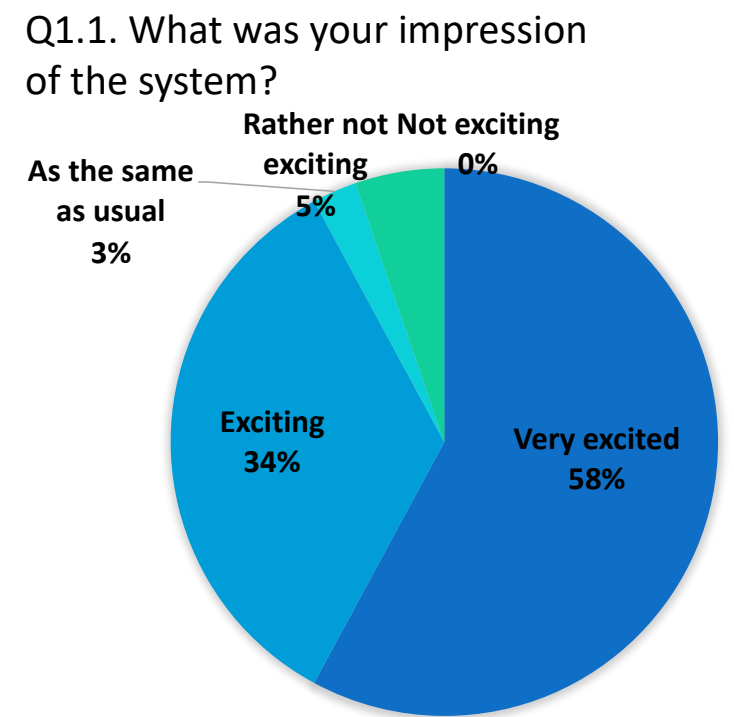

Q1.3. Did you understand the intermolecular force?

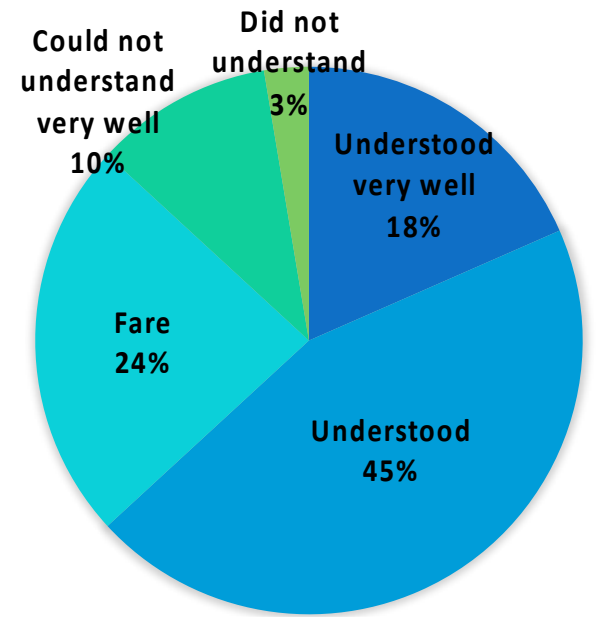

Q1.2. Did you have enough time to conduct the experiment?

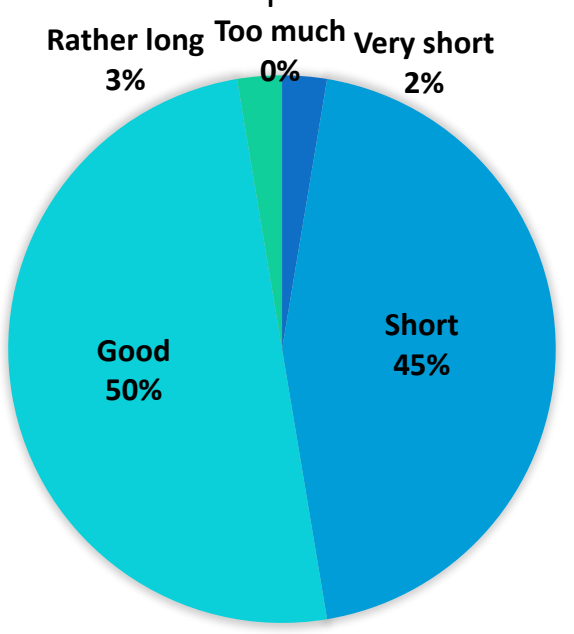

Q1.4. Do you expect to use this system in the future class too? Rather not No, it is not

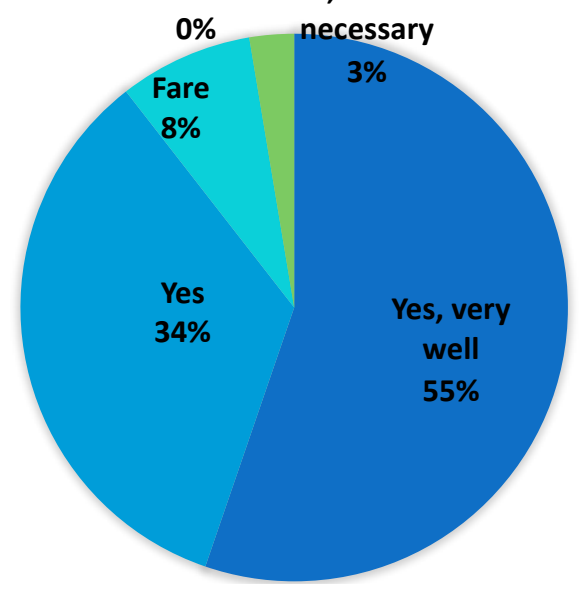

Figure 6. Answers to questionaries on the lecture 
For questionnaires on the HaptiChem equipment, about $95 \%$ of the students did not find it difficult to operate the system (Figure 7-1), and about 76\% did not worry about the stability of the system while using it (Figure 7-2), even though the teacher cautioned them to handle the system very carefully before the experiments, because the haptic device was valuable. About $92 \%$ of the students easily understood what the display meant (Figure 7-3). About 95\% thought that they needed the HaptiChem system in places where they could easily access it, such as at their homes, in their classes, and high-school (Figure 7-4).
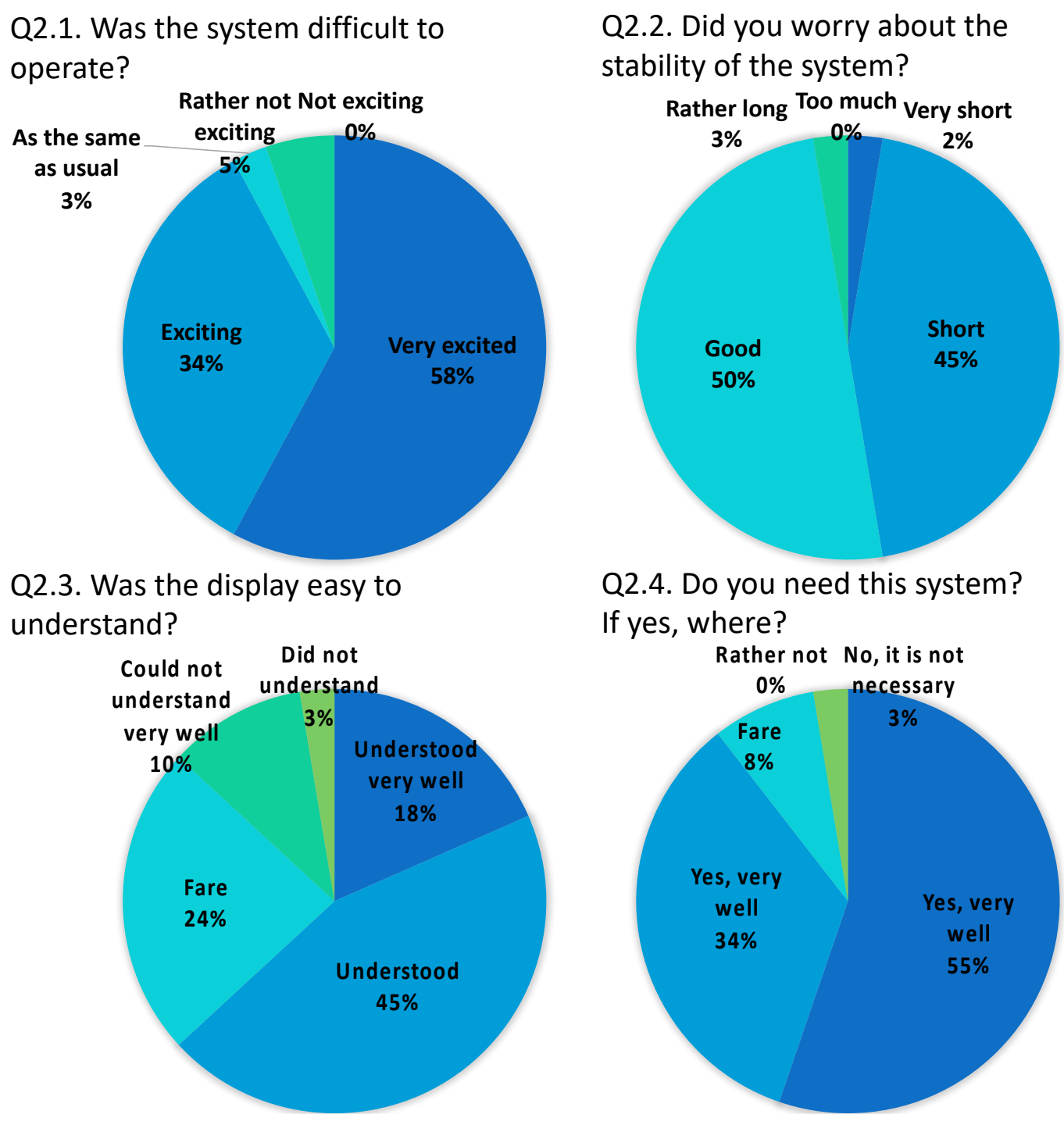

If yes, where?

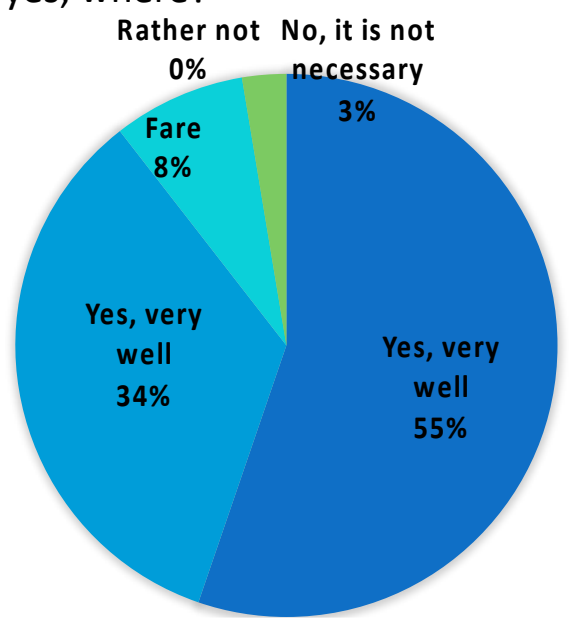

Figure 7. Answers to questionaries on the HaptiChem device

In the questionnaire free writing space for both the lecture and system, 24 students wrote what they liked. Most of them made positive comments expressing their excited feelings gained from the system, for example, "I was really surprised, because the system was extraordinarily exciting and more robust than I expected.", "I think that it must be more interesting if we can feel the intermolecular force between bigger molecules.", "I guess that I could better understand the contents of the lecture by actually feeling the force.", "I grasped the meaning of the potential 
and force function chart more deeply by actually feeling the changes of the force.", "I would like to buy the system for myself.", and "This system is exciting! I am interested in the development of this system in future, too." Some of the students seemed to be interested in the mechanism of the haptic device.

These results show that the impression of the students was totally positive and implies that they learned with scientific curiosity and interests. The answers to the questionnaires on the system indicated that they evaluated the stability and usability of HaptiChem as sufficient.

\section{Conclusion}

We reported on our first attempt at the application of the HaptiChem system to chemical education. The chemistry class that used the system was stimulated into thinking more deeply about and widely imagining science and encouraged to study with more curiosity. These are the results from students who basically liked chemistry, but sufficiently demonstrated the effectiveness of the HaptiChem system in chemical education.

This is a report of a first pilot experiment with a simple haptic system. More detailed experiments and analyses are needed to quantitatively measure the effectiveness of haptic systems in education. Such further investigations should be possible in the modern age by using more effective tools developed with the modern technologies. Since the first trial class in 2007, HaptiChem has been regularly used in chemistry classes in the high school with the same students' positive reactions. We believe that educational tools using VR technology will become common in the future that provide students with opportunities to become more interested in and curious about chemistry.

\section{Acknowledgements}

The experiment could be successfully conducted thanks to the kind cooperation by the teachers and staffs at Tokyo Tech High School of Science and Technology. Especially we would like to sincerely appreciate Mr. Masaru Moriyasu, a former teacher of chemistry at the high school. He held the lecture in the chemistry class. The plan of the class could not be implemented without his efforts and deep understanding of the effectiveness of a haptic molecular system for chemical education. We would like to say great thanks to Ms. Hiroko Asaoka. She was one of the main developers of HaptiChem software when she was a staff at SRA Key Technology Laboratory, Inc., Tokyo. We appreciate her sincere and steady work. This work was supported in part by a Grantin-Aid for Scientific Research on Priority Area (A) 17011076 from the Ministry of Education, Culture, Sports, Science and Technology (MEXT), Japan, to H. Satoh and by the Funding for Joint Research from the National Institute of Informatics to H. Satoh., K. Akahane., and M. Sato. 


\section{References}

1. F. P. Brooks, M. Ouh-Young, J. J. Batter, P. J. Kilpatrick, Project GROPE-Haptic Displays for Scientific Visualization, Computer Graphics, 24(4), 177-185 (1990)

2. D. Levine, M. Facello, P. Hallstrom, G. Reeder, B. Walenz, F. Stevens, An Interactive System for Virtual Molecular Docking, IEEE Computational Science \& Engineering, 4(2), 55-65 (1997)

3. E. Harvey, C. Gingold, Haptic Representation of the Atom, The Proceedings of the IEEE International Conference on Information Visualization (IV'00), 232-234 (2000)

4. J. E. Stone, J. Gullingsrud, K. Schulten, A System for Interactive Molecular Dynamics Simulation, Proceedings of the Symposium on Interactive 3D Graphics, 191-194 (2001)

5. G. Sankaranarayanan, S. Weghorst, M. Sanner, A. Gillet, A. Olson, Role of Haptics in Teaching Structural Molecular Biology, Proceedings of the $11^{\text {th }}$ Symposium on Haptic Interfaces for Virtual Environment and Teleoperator Systems (HAPTICS'03), 363-366 (2003)

6. Y. G. Lee, K. W. Lyons, Smoothing Haptic Interaction Using Molecular Force Calculations, Computer-Aided Design, 36, 75-90 (2004)

7. R. A. Davies, N. W. John, J. N. MacDonald, K. H. Hughes, Visualization of Molecular Quantum Dinamics - A Molecular Visualization Tool with Integrated Web3D and Haptics, Web3D Symposium Proceedings, 143-150 (2005)

8. H. Satoh, T. Nukada, K. Akahane, M. Sato, Construction of Basic Haptic Systems for Feeling the Intermolecular Force in Molecular Models, J. Comput. Aided Chem., 7, 38-47 (2006)

9. K. H. Marti, M. Reiher, Haptic Quantum Chemistry, J. Comput. Chem. 30, 2010-2020 (2009)

10. M. P. Haag, K. H. Marti, M. Reiher, Generation of Potential Energy Surfaces in High Dimensions and Their Haptic Exploration, ChemPhysChem, 12, 3204-3213 (2011)

11. A. Ricci, A. Anthopoulos, A. Massarotti, I. Grimstead, A. Brancale, Haptic-driven Applications to Molecular Modeling: State-of-the-art and Perspectives, Future Medicinal Chemistry, 10.4155/fmc.12.60, 4, 10, 1219-1228, (2012)

12. M. P. Haag, A. C. Vaucher, M. Bosson, S. Redon, M. Reiher, Interactive Chemical Reactivity Exploration, ChemPhysChem, 15, 3301-3319 (2014)

13. Moritz P. Haag, Markus Reiher, Studying chemical reactivity in a virtual environment, Faraday Discuss., 10.1039/C4FD00021H, 169, 89-118, (2014)

14. N. Luehr, A. G. B. Jin, T. J. Martínez, Ab Initio Interactive Molecular Dynamics on Graphical Processing Units (GPUs) , J. Chem. Theory Comput. 11, 10, 4536-4544, (2015)

15. A. C. Vaucher, M. P. Haag, M. Reiher, Real-time Feedback from Interactive Electronic Structure Calculations, J. Comp. Chem. 37, 805-812 (2016)

16. http://www.sensable.com/products/phantom_ghost/phantom.asp

17. M. Sato, Y. Hirata, H. Kawarada, Rotating Shape Modeling with SPIDAR" Journal of Robotics and Mechatronics, 4, 31-38 (1992)

18. M. Martino, A. Salvadori, F. Lazzari, L. Paoloni, S. Nandi, G. Mancini, V. Barone, S. Rampino, Chemical promenades: Exploring potential-energy surfaces with immersive virtual reality, J. Comput. Chem., 41, 13, 1310-1323, (2020)

19. H. M. Deeks, R. K. Walters, S. R. Hare, M. B. O'Connor, A. J. Mulholland, D. R. Glowacki, Interactive Molecular Dynamics in Virtual Reality for Accurate Flexible Protein-Ligagnd Docking, PLOS ONE, 15(3), e0228461 (2020)

20. http://arachnoforce.com/spidar-w.html, accessed 5 October 2021.

21. D. J. Lipomi, C. Dhong, C. W. Carpenter, N. B. Root, V. S. Ramachandran, Organic Haptics: Intersection of Materials Chemistry and Tactile Perception, Adv. Funct. Mater. 30, 1906850 (2020)

22. K. Nakakoji, H. Satoh, Y. Yamamoto, A. Aoki, H. Asaoka, Interaction Design for Molecular Reaction Prediction. IPSJ SIG Technical Report, 2003-HI-106, 2003-MUS-52, 79-86 (2003)

23. K. Nakakoji, H. Satoh, Toward Interaction Design for Molecular Visualization: Matching Operation Primitives with Devices. IPSJ SIG Technical Report, 2005-HI-113, 1-7(2005) 
24. H. Satoh, HaptiChem: Haptic Molecular Modeling System and ChemoJun: Chemical Graphics Library for Chemical Education. 54th Meeting of High Schools of Tokyo Area, Tokyo, Japan, July, 2006.

25. H. Satoh, A. Aoki, H. Asaoka, Development of Software for Visualizing and Force-Display of Chemical Information. The 19th International Conference on Chemical Education, Seoul, Korea, August, 2006.

26. H. Satoh, M. Sato, HaptiChem: Intermolecular Force Display System Using Haptic Device SPIDAR-G. Gordon Research Conference on Visualization in Science \& Education, Smithfield, USA, July, 2007.

27. https://www.itmedia.co.jp/news/articles/0703/15/news104.html, accessed 5 October 2021.

28. https://xtech.nikkei.com/it/article/NEWS/20070315/265391/, accessed 5 October 2021.

29. http://techon.nikkeibp.co.jp/article/NEWS/20070319/129070/, accessed 5 October 2021.

30. https://www.ccsnews.jp/ccs2/2007/1q/2007_1Qniisatohaptichem.htm, accessed 5 October 2021.

31. https://www.hst.titech.ac.jp/NL/Vol3/NL_vol3_no12.html, accessed 5 October 2021.

32. M. Sato, X. Liu, J. Murayama, K. Akahane, M. Isshiki, A Haptic Virtual Environment for Molecular Chemistry Education. T. Edutainment. 1. 28-39 (2008)

33. S. Comai, D. Mazza, A Haptic-Based Framework for Chemistry Education: Experiencing Molecular Interactions with Touch. In: Lytras M.D. et al. (eds) Technology Enhanced Learning. Quality of Teaching and Educational Reform. TECH-EDUCATION 2010. Communications in Computer and Information Science, vol 73. Springer, Berlin, Heidelberg (2010)

34. B. I. Edwards, K. S. Bielawski, R. F. Prada, A. D. Cheok, Haptic Virtual Reality and Immersive Learning for Enhanced Organic Chemistry Instruction. Preprints, 2018040065 (2018) (doi: 10.20944/preprints201804.0065.v1)

35. S. Kim, S. Hasegawa, Y. Koike, M. Sato, Tension based 7-DOF force feedback device: SPIDAR-G. Proceedings IEEE Virtual Reality 2002, 2002, pp. 283-284, doi: 10.1109/VR.2002.996540.

36. https://youtu.be/RsqblzUxmg8, accessed 5 October 2021.

37. http://arachnoforce.com/spidar-g.html, accessed 5 October 2021.

38. http://arachnoforce.com/movie.html, accessed 5 October 2021.

39. H. Satoh, A. Aoki, H. Asaoka, ChemoJun: Open Source Chemical Graphics Library, J. Comput. Aided Chem., 7, 141-149 (2006).

40. https://github.com/shiroco/ChemoJun, accessed 5 October 2021.

41. https://www.cincomsmalltalk.com/main/community/product-portal/contributed/jun/, accessed 5 October 2021.

42. http://research.nii.ac.jp/\%7Echeminfo/HaptiChem/index.html, accessed 5 October 2021.

43. https://github.com/shiroco/HaptiChem, accessed 5 October 2021.

44. Cincom Systems Headquarters (https://www.cincomsmalltalk.com/main/) and Cincom Systems Japan (https://www.cincom.co.jp), accessed 5 October 2021. 